\title{
Produção, qualidade e conservação de tomates heterozigotos nos locos alcobaça, nonripening e ripening inhibitor
}

\author{
Alcides Militão dos Santos Júnior(1), Wilson Roberto Maluf(2), Marcos Ventura Faria( ${ }^{(3)}$, \\ Valter Carvalho de Andrade Júnior ${ }^{(4)}$, Ildon Rodrigues do Nascimento(5), Flávio Rodrigo Gandolfi Benites ${ }^{(5)}$ \\ e Luiz Antônio Augusto Gomes ${ }^{(2)}$
}

\begin{abstract}
(1)Centro Federal de Educação Tecnológica de Petrolina, Rod. BR 235, Km 22, CEP 56302-970 Petrolina, PE (2)Universidade Federal de Lavras (UFLA), Dep. de Agricultura, Caixa Postal 35, CEP 37200-000 Lavras, MG. E-mail: wrmaluf@ufla.br (3)Universidade Estadual do CentroOeste, Dep. de Agronomia, Centro Politécnico, Rua Simeão Camargo Varela de Sá, o 3, CEP 85040-080 Guarapuava, PR. E-mail: mfaria@unicentro.br (4)Faculdades Federais Integradas de Diamantina, Dep. de Agronomia, Rua da Glória, no 187, Campus I, CEP $39100-000$ Diamantina, MG. E-mail: valterj@@fafeid.edu.br (5)UFLA, Dep. de Biologia. E-mail: nascimento_ildon@yahoo.com.br, frgbenites@yahoo.com.br
\end{abstract}

Resumo - O objetivo deste trabalho foi avaliar os atributos de produtividade, qualidade e conservação póscolheita de tomates, para comparar os efeitos promovidos pelos alelos alcobaça $\left(\right.$ nor $\left.^{4}\right)$, nonripening (nor) e ripening inhibitor (rin) em heterozigose, isoladamente ou em duplas combinações, sobre frutos de tomateiros híbridos. Foram avaliados dez tratamentos: sete híbridos experimentais quase-isogênicos, com background

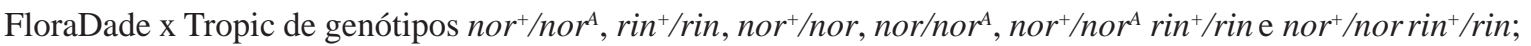
e três testemunhas comerciais (Floradade, Tropic e Carmen $\mathrm{F}_{1}$ ). Contrariamente aos genótipos $\mathrm{rin}^{+} / \mathrm{rin}$ e nor $^{+} /$ nor, o genótipo nor $^{+} /$nor $^{A}$ não prolongou, significativamente, a firmeza dos frutos em pós-colheita. Os genótipos

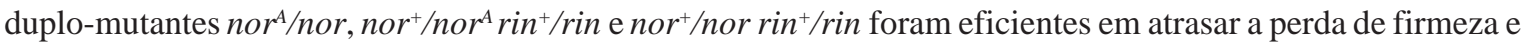
a evolução da coloração dos frutos; os efeitos dos locos nor $/$ nor $^{A}$ e rin $^{+} / r i n$, juntos, sofreram desvios significativos em relação à soma dos efeitos desses locos, quando atuaram separadamente, no sentido de intensificarem esses atrasos. O uso de híbridos heterozigotos, nas duplas combinações entre os locos nor ${ }^{A}$, nor e rin, mostrou-se vantajoso por propiciar frutos firmes, com maior extensão da vida pós-colheita, em comparação com o uso dos híbridos portadores desses locos isoladamente. A qualidade dos frutos duplo-mutantes não foi limitada pelo atraso na evolução da coloração vermelha.

Termos para indexação: Lycopersicon esculentum, firmeza de frutos, melhoramento genético, mutantes de amadurecimento, tomate longa-vida.

\section{Yield, quality and conservation of heterozygous tomatoes in alcobaça, nonripening and ripening inhibitor loci}

\begin{abstract}
Thie objective of this work was to evaluate yield, quality and postharvest conservation of tomatoes, to compare the effects promoted by the alcobaça $\left(\right.$ nor $\left.^{A}\right)$, nonripening (nor) and ripening inhibitor (rin) alleles, in heterozygosis, singly or in double combinations on hybrid tomato fruits. Ten treatments were evaluated: seven experimental nearly isogenic hybrids of FloraDade x Tropic background or reciprocal with nor $/ \mathrm{nor}^{\mathrm{A}}, \mathrm{rin}^{+} /$ rin, nor ${ }^{+} /$nor, nor $/$nor $^{A}$, nor ${ }^{+} /$nor $^{A}$ rin $^{+} /$rin and nor $/$nor rinn $^{+} /$rin genotypes, and three commercial checks (FloraDade, Tropic and Carmen $\mathrm{F}_{1}$ ). Oppositely to the genotypes $\mathrm{rin}^{+} / \mathrm{rin}$ and nor $^{+} /$nor, the genotype nor $^{+} /$nor $^{A}$ did not significantly extend the firmness of the fruits in postharvest. The double mutant genotypes nor ${ }^{A} /$ nor, nor $^{+} /$nor $^{A}$ rin $^{+} /$rin and nor $/$nor rin $^{+} /$rin were efficient in delaying firmness loss and the evolution of fruits colouring; the effects of loci nor ${ }^{+} /$or $^{A}$ and $\mathrm{rin}^{+} /$rin, together, underwent significant deviations relatively to the sum of these loci effects, when acting singly, in the sense of enhancing those delays. Use of heterozygous hybrids in the double combinations among loci nor ${ }^{A}$, nor and rin proved advantageous for providing firm fruits with a longer postharvest lifetime, as compared with the hybrids carrying these loci singly. The quality of double mutant fruits was not restricted by the delay in the evolution of red coloration.
\end{abstract}

Index terms: Lycopersicon esculentum, fruit firmness, plant breeding, ripening mutants, long shelf life tomato. 


\section{Introdução}

Uma estratégia para prolongar a vida pós-colheita dos frutos de tomate é o emprego de alelos mutantes de maturação, que retardam o amadurecimento, prolongam o período de conservação e interferem, principalmente, na firmeza e na síntese de pigmentos carotenóides (Kopeliovitch et al., 1979; Araújo et al., 2002; Santos Junior, 2002; Andrade-Júnior, 2003; Faria et al., 2003).

$\mathrm{O}$ mutante ripening inhibitor (rin) localiza-se no cromossomo 5, e os mutantes nonripening (nor) e alcobaça $\left(\right.$ nor $\left.^{A}\right)$ localizam-se no cromossomo 10 , do genoma do tomateiro; rin está ligado em atração ao alelo macrocalyx $(m c)$, que confere a formação de um cálice maior, enquanto nor está ligado, em repulsão, a 3,5 cM do alelo uniform ripening $(u)$, que confere maturação uniforme dos frutos (Tigchelaar et al., 1978). Ambos os mutantes promovem redução na síntese de carotenóides (Sink et al., 1974; Tigchelaar et al., 1978), retardam o amolecimento e aumentam a conservação pós-colheita dos frutos. Entretanto, em heterozigose, tais efeitos diminuem (Buescher \& Tigchelaar, 1975; Tigchelaar et al., 1978; Kopeliovitch et al., 1979). Segundo Tigchelaar et al. (1978), os frutos de tomateiros mutantes homozigotos rin/rin apresentam amadurecimento do tipo não-climatérico, o que implica a paralisação do amadurecimento, quando colhidos na fase inicial de maturação, e a ausência de resposta à aplicação de etileno exógeno.

O mutante alcobaça controla um padrão atípico de amadurecimento nos frutos (Lobo, 1981; Lobo et al., 1984; Mutschler, 1984a, 1984b), retarda o amolecimento e permite o aumento do período de armazenamento (Lobo, 1981; Mutschler, 1984b; Freitas et al., 1998; Vilas Boas et al., 1999; Araújo et al., 2002; Faria et al., 2003). Testes de alelismo realizados por Lobo (1981) e confirmados por Benites (2003), demonstraram que alcobaça é um mutante não alélico a rin, porém, alélico a nor, constitui-se num terceiro alelo do loco nor e recebe a denominação de $n o r^{A}$.

Os relatos na literatura que descrevem e comparam os efeitos dos alelos nor ${ }^{A}$, nor e rin, em atuação conjunta, em um mesmo genótipo, são poucos e recentes (Santos Junior, 2002; Andrade-Júnior, 2003; Benites, 2003).

Este trabalho teve como objetivo avaliar os efeitos das combinações genotípicas heterozigotas, entre os alelos nor ${ }^{A}$, nor e rin, sobre a produção e a qualidade pós-colheita de frutos de tomateiro, visando o desenvolvimento de híbridos com prolongada vida de prateleira.

\section{Material e Métodos}

Foram obtidos sete híbridos experimentais de tomateiro de background FloraDade $\mathrm{x}$ Tropic ou recíproco, a partir de cruzamentos entre linhagens utilizadas como genitores femininos [FloraDade $\left(\mathrm{nor}^{+} / \mathrm{nor}^{+} \mathrm{rin}^{+} / \mathrm{rin}^{+}\right)$, TOM-613 (nor/nor rin $^{+} /$rin $^{+}$), TOM-610 (nor ${ }^{A} /$ nor $^{A}$ $\left.\mathrm{rin}^{+} / \mathrm{rin}^{+}\right)$e TOM-619 (nor ${ }^{+} /$nor $^{+}$rin/rin)] e linhagens utilizadas como fonte de pólen [Tropic (nor ${ }^{+} / \mathrm{nor}^{+} \mathrm{rin}^{+} /$ $\left.\mathrm{rin}^{+}\right)$, FloraDade $\left(\right.$nor $\left.^{+} / \mathrm{nor}^{+} \mathrm{rin}^{+} / \mathrm{rin}^{+}\right)$, TOM-610 $\left(\right.$ nor $^{\mathrm{A}} /$ nor $\left.^{\mathrm{A}} \mathrm{rin}^{+} / \mathrm{rin}^{+}\right)$, TOM-559 $\left(\right.$nor $^{\mathrm{A}} /$ nor $\left.^{\mathrm{A}} \mathrm{rin}^{+} / \mathrm{rin}^{+}\right)$ e TOM-613 (nor/nor $\left.\mathrm{rin}^{+} / \mathrm{rin}^{+}\right)$]. Os sete híbridos representam, além do background normal [ $\mathrm{F}_{1}$ (FloraDade $\mathrm{x}$ Tropic)], suas versões quase-isogênicas nor $^{+} /$nor $^{A}$, nor ${ }^{+} /$nor, rin $^{+} /$rin, nor $/$/nor, nor ${ }^{+} /$nor $^{A}$ rin $^{+} /$rin e nor $^{+} /$ nor $\mathrm{rin}^{+} /$rin. Os sete híbridos experimentais, juntamente com as linhagens parentais FloraDade e Tropic, mais o híbrido comercial Carmen $\mathrm{F}_{1}\left(\mathrm{rin}^{+} /\right.$rin) constituíram os dez tratamentos avaliados.

O experimento foi conduzido em estufa de plástico, na HortiAgro Sementes Ltda., no Município de Ijaci, MG, de dezembro de 2000 a abril de 2001. Foi empregado o delineamento em blocos ao acaso, com seis repetições. Cada parcela foi composta de uma fileira com oito plantas espaçadas em $0,8 \mathrm{~m}$. As plantas foram conduzidas com uma haste e podadas acima do quinto cacho. Na ocasião da antese, as flores foram marcadas, com a finalidade de se determinar o número de dias entre a antese e o ponto de colheita, no estádio breaker de maturação, determinado pelo início de coloração avermelhada na região da cicatriz estilar do fruto. Foram realizadas 16 colheitas durante um período de 38 dias. Avaliou-se a produção total $\left(\mathrm{t} \mathrm{ha} \mathrm{H}^{-1}\right)$, produção precoce (produção das cinco primeiras colheitas, em $\mathrm{t} \mathrm{ha}^{-1}$ ), massa média por fruto (g por fruto), e o número médio de dias desde a antese ao estádio breaker de maturação (ponto de colheita).

Amostras de oito frutos, por parcela, foram colhidas no estádio breaker e armazenadas em prateleiras, em sala com ambiente controlado $\left(15^{\circ} \mathrm{C}\right.$ e $60 \%$ de umidade relativa), na Universidade Federal de Lavras. Os frutos foram avaliados quanto à taxa de perda de firmeza e evolução da coloração externa vermelha, durante o período de armazenamento. A firmeza $\left(\mathrm{N} \mathrm{m}^{-2}\right)$ foi medida na superfície equatorial de cada fruto, individualmente, 
por meio da técnica de aplanação não-destrutiva, descrita por Calbo \& Nery (1995). As leituras da firmeza foram obtidas no estádio breaker de amadurecimento (dia zero) e a intervalos de dois dias, até o 18ํ dia após a colheita. Os mesmos frutos amostrados receberam notas, diariamente, com base em uma escala que variou de 1 a 5, conforme a porcentagem de coloração vermelha na superfície de cada fruto. A porcentagem de coloração da superfície, no dia zero (estádio breaker), recebeu a nota 1 . As notas 2, 3, 4 e 5 corresponderam às porcentagens de coloração da superfície dos frutos entre $20 \%$ e $40 \%$, $41 \%$ e $60 \%$, $61 \%$ e $80 \%$ e $81 \%$ e $100 \%$, respectivamente.

Foram ajustados modelos de regressão não-linear para a taxa de perda de firmeza e do desenvolvimento da coloração vermelha externa dos frutos, durante o período de armazenamento. O comportamento da perda da firmeza dos frutos foi ajustado a um modelo de decaimento exponencial, que foi empregado para se estimar a firmeza inicial dos frutos (no estádio breaker), a meia-vida da firmeza (tempo em pós-colheita que o fruto leva para reduzir à metade sua firmeza, em relação à inicial) e o número de dias decorridos para os frutos atingirem a firmeza crítica de $2.10^{4} \mathrm{~N} \mathrm{~m}^{-2}$.

A meia-vida de firmeza (T) foi obtida por meio da regressão dos dados de firmeza (A) de cada parcela, no número de dias decorridos $(\mathrm{X})$, pelo modelo estatístico: $\mathrm{A}=\mathrm{A}_{0}(1 / 2)^{\mathrm{X} / \mathrm{T}}$, em que $\mathrm{A}_{0}$ é a firmeza $\left(\mathrm{N} \mathrm{m}^{-2}\right)$ inicial dos frutos, no estádio breaker (dia 0); X é o número de dias decorridos após colheita, no estádio breaker; $\mathrm{T}$ é a meia-vida da firmeza (medida em dias); A é a firmeza $\left(\mathrm{N} \mathrm{m}^{-2}\right)$ depois de $\mathrm{X}$ dias. As regressões foram calculadas com o recurso do pacote estatístico SAS (Statistical Analysis System), utilizando-se, para o modelo de decaimento exponencial, a transformação logarítmica seguida de regressão linear.

$\mathrm{O}$ modelo logístico $\mathrm{Y}=\mathrm{A} /\left(1+\mathrm{B} \times \mathrm{R}^{\mathrm{X}}\right)$ foi ajustado às notas da evolução da coloração vermelha externa dos frutos ( $\mathrm{X}$ é número de dias após a colheita; $\mathrm{Y}$ é a nota de coloração dos frutos; A, B e R são parâmetros do modelo). O número médio de dias decorridos a partir da colheita, para que os frutos atingissem a nota 5 de coloração, foi estimado a partir da curva ajustada.

Amostras adicionais de frutos, de cada parcela, foram empregadas para a quantificação dos teores de clorofila total e pigmentos carotenóides, em três diferentes estádios de maturação: breaker, intermediário e com- pletamente maduro, correspondentes às notas externas de coloração 1 , 3 e 5, respectivamente. Os teores de clorofila total foram determinados segundo Bruinsma (1963), e os resultados foram expressos em mg por $100 \mathrm{~g}$ de polpa. A quantificação dos teores de pigmentos carotenóides (licopeno e beta-caroteno), expressos em mg por 100 g de polpa, foi determinada segundo Nagata \& Yamashita (1992). Os valores médios, referentes aos teores de pigmentos carotenóides e clorofila, foram submetidos à análise de variância e as médias foram comparadas pelo teste de Tukey.

Para todos os caracteres, foram calculados contrastes não-ortogonais de interesse, entre os pares de híbridos, para avaliar os efeitos isolados dos alelos nor, nor $^{A}$ e rin, em heterozigose, bem como os efeitos das duplas combinações heterozigóticas entre esses locos. Foram calculados, também, contrastes para avaliar a nãoaditividade entre os efeitos dos locos em questão, dois a dois. A não-aditividade dos efeitos de cada dois alelos mutantes, em heterozigose, corresponde ao desvio do genótipo portador da dupla combinação entre esses alelos, relativamente à soma dos seus efeitos isoladamente.

\section{Resultados e Discussão}

Entre os híbridos de background FloraDade x Tropic, o de genótipo $\mathrm{rin}^{+} /$rin apresentou redução significativa da produção total de frutos. Houve efeito negativo do alelo nor, sobre a produção total de frutos, somente em combinações com nor ${ }^{A}$ ou rin heterozigotos (Tabela 1). O genótipo nor $^{+} /$nor $^{A}$ não teve a produtividade total de frutos afetada, o que concorda com os resultados relatados por Freitas et al. (1998) e Faria et al. (2003) em híbridos com backgrounds distintos.

Os genótipos nor ${ }^{+} /$nor $^{A}$, rin $^{+} /$rin e nor $/$nor apresentaram redução significativa na produção precoce, no background FloraDade x Tropic (Tabela 1), tendo refletido o efeito dos alelos mutantes, mesmo em heterozigose, no sentido de retardar o início do estádio breaker. No entanto, a produção precoce é afetada não somente pelos mutantes de amadurecimento, mas também pelo background, uma vez que o híbrido comercial Carmen $\mathrm{F}_{1}\left(\mathrm{rin}^{+} /\right.$rin) obteve maior produção precoce do que o híbrido experimental rin $^{+} /$rin $\mathrm{F}_{1}$ (TOM-619 x FloraDade) (Tabela 1). 
Os genótipos nor ${ }^{+} /$nor e nor $^{+} /$nor $^{A} \mathrm{rin}^{+} / \mathrm{rin}$ atuaram no sentido de reduzir ligeiramente a massa média de frutos, em relação ao genótipo normal (Tabela 1). $\mathrm{O}$ alelo nor $^{A}$ em heterozigose não afetou a massa média de frutos, o que concorda com os resultados encontrados por Mutschler (1992), Freitas et al. (1998), Souza et al. (2001), Dias et al. (2003) e Faria et al. (2003). Da mesma forma, os frutos de genótipo $\mathrm{rin}^{+} /$rin não tiveram massa média alterada em relação ao genótipo normal (Tabela 1). Foi verificada ausência de heterose para maior peso de frutos, concordando com o que tem sido relatado por outros autores (Maluf et al., 1982; Faria et al., 2003).

Não foi detectado efeito significativo do genótipo nor $^{+} /$nor $^{A}$ sobre o período de permanência dos frutos na planta até o estádio breaker; resultado semelhante foi relatado por Faria et al. (2003). Os alelos nor e rin, isoladamente em heterozigose, não afetaram essa característica, entretanto, quando em duplas combinações heterozigotas $\left(\right.$ nor ${ }^{\mathrm{A}} /$ nor, nor $^{+} /$nor $^{\mathrm{A}} \mathrm{rin}^{+} /$rin e nor $^{+} /$nor $\mathrm{rin}^{+} /$rin) promoveram aumento significativo na permanência dos frutos na planta em 3,5 dias, 2,2 dias e 2,4 dias, respectivamente (Tabela 1 ). O contraste que mede a não-aditividade entre os alelos nor e nor $^{A}$ foi significativo. Portanto, esses alelos, juntos no mesmo genótipo (nor ${ }^{A} /$ nor), atuaram de forma mais acentuada, aumentando em cerca de três dias o período da antese ao estádio breaker de maturação dos frutos, em relação à soma dos seus efeitos individuais (Tabela 1).

Não houve diferenças significativas entre os híbridos experimentais quanto à firmeza inicial dos frutos (Tabela 2). A meia-vida da firmeza dos frutos foi aumentada de modo significativo, em 1,6 e 2,2 dias, respectivamente, pelos mutantes de amadurecimento nor e rin, em heterozigose, que atuaram isoladamente (Tabela 2). As duplas combinações heterozigóticas entre os alelos nor ${ }^{A}$, nor e rin atuaram no sentido de prolongar a meiavida da firmeza dos frutos, em relação aos efeitos individuais dos alelos em heterozigose. Os frutos de genótipos nor ${ }^{A} /$ nor, nor ${ }^{+} /$nor $^{A}$ rin $^{+} /$rin e nor ${ }^{+} /$nor rin $^{+} /$ rin tiveram suas meias-vidas aumentadas em 2,4 dias, 3,3 dias e 3,4 dias, respectivamente, em comparação aos frutos normais (Tabela 2). Os contrastes que medem a

Tabela 1. Valores médios da produção total $\left(\mathrm{t} \mathrm{ha} \mathrm{a}^{-1}\right)$, produção precoce $(\mathrm{t}$ ha-1), massa média por fruto (g por fruto), período médio da antese ao estádio breaker (dias) e contrastes não-ortogonais de interesse entre dez genótipos de tomateiro(1).

\begin{tabular}{|c|c|c|c|c|c|}
\hline Tratamento & Genótipo & $\begin{array}{c}\text { Produção } \\
\text { total }\end{array}$ & $\begin{array}{c}\text { Produção } \\
\text { precoce }\end{array}$ & $\begin{array}{c}\text { Massa } \\
\text { por fruto }\end{array}$ & $\begin{array}{l}\text { Período da antese } \\
\text { ao estádio breaker }\end{array}$ \\
\hline T1 - FloraDade & nor $^{+} /$nor $^{+}$rin $^{+} /$rin $^{+}(=$normal $)$ & $65,61 \mathrm{ab}$ & $16,28 b$ & $169,63 \mathrm{~cd}$ & $44,97 \mathrm{de}$ \\
\hline T2 - Tropic & nor $^{+} /$nor $^{+} \operatorname{rin}^{+} / \operatorname{rin}^{+}(=$normal $)$ & $65,74 \mathrm{ab}$ & $13,85 b$ & $225,95 \mathrm{a}$ & $47,68 \mathrm{abc}$ \\
\hline $\mathrm{T} 3-\mathrm{F}_{1}$ (FloraDade $\mathrm{x}$ Tropic) & nor $^{+} /$nor $^{+} \mathrm{rin}^{+} / \mathrm{rin}^{+}(=$normal $)$ & $65,68 \mathrm{ab}$ & $17,18 b$ & $211,95 \mathrm{ab}$ & $45,38 \mathrm{cde}$ \\
\hline $\mathrm{T} 4-\mathrm{F}_{1}$ (TOM $-610 \times$ FloraDade $)$ & nor $^{+} /$or $^{A} \mathrm{rin}^{+} / \mathrm{rin}^{+}$ & $63,11 \mathrm{ab}$ & $12,05 \mathrm{bc}$ & $192,62 \mathrm{abc}$ & $45,12 \mathrm{de}$ \\
\hline T5 - $\mathrm{F}_{1}$ (TOM -613 x Tropic) & nor $/$ nor $\operatorname{rin}^{+} /$rin $^{+}$ & $58,62 b$ & $11,90 \mathrm{bc}$ & $189,77 \mathrm{bc}$ & 46,13 bcde \\
\hline T6 - $\mathrm{F}_{1}(\mathrm{TOM}-619 \times$ FloraDade $)$ & nor $^{+} /$nor $^{+}$rin $^{+} /$rin & $46,96 b$ & $6,34 \mathrm{~cd}$ & $192,55 \mathrm{abc}$ & $46,72 \mathrm{abcd}$ \\
\hline T7 $-\mathrm{F}_{1}(\mathrm{TOM}-613 \times \mathrm{TOM}-610)$ & nor ${ }^{A} /$ nor $\mathrm{rin}^{+} / \mathrm{rin}^{+}$ & $45,46 b$ & $3,37 \mathrm{~d}$ & $197,83 \mathrm{abc}$ & $48,88 \mathrm{a}$ \\
\hline T8 - $\mathrm{F}_{1}(\mathrm{TOM}-619$ х TOM -559$)$ & nor $^{+} /$nor $^{A} \operatorname{rin}^{+} /$rin & $59,64 \mathrm{~b}$ & $5,63 \mathrm{~cd}$ & $187,48 \mathrm{bc}$ & $47,60 \mathrm{abc}$ \\
\hline T9 $-\mathrm{F}_{1}(\mathrm{TOM}-619 \times \mathrm{TOM}-613)$ & nor $/$ nor $\mathrm{rin}^{+} /$rin & $48,85 b$ & $5,46 \mathrm{~cd}$ & $191,35 \mathrm{abc}$ & $47,77 \mathrm{ab}$ \\
\hline T10 - Carmen F 1 & nor $^{+} /$nor $^{+}$rin $^{+} /$rin & $83,51 \mathrm{a}$ & $24,74 \mathrm{a}$ & $143,90 \mathrm{~d}$ & $44,38 \mathrm{e}$ \\
\hline Contraste de interesse & Efeito avaliado & \multicolumn{4}{|c|}{-------------- Estimativas dos contrastes ----------- } \\
\hline $\mathrm{T} 1-\mathrm{T} 2$ & FloraDade $\mathrm{x}$ Tropic & $-0,135$ & 2,425 & $-56,317^{* *}$ & $-2,72^{* *}$ \\
\hline $\mathrm{T} 3-[0,5(\mathrm{~T} 1+\mathrm{T} 2)]$ & Heterose no background normal & 0,008 & 2,093 & 14,158 & $-0,94$ \\
\hline $\mathrm{T} 4-\mathrm{T} 3$ & Apenas nor $^{+} /$nor $^{A}$ & $-2,570$ & $-5,108^{*}$ & $-19,333$ & $-0,27$ \\
\hline $\mathrm{T} 5-\mathrm{T} 3$ & Apenas nor $^{+} /$nor & $-7,058$ & $-5,255^{*}$ & $-22,183^{*}$ & 0,75 \\
\hline $\mathrm{T} 6-\mathrm{T} 3$ & Apenas $\mathrm{rin}^{+} / \mathrm{rin}$ & $-18,722^{* *}$ & $-10,815^{* *}$ & $-19,400$ & 1,33 \\
\hline $\mathrm{T} 7-\mathrm{T} 3$ & Dupla combinação nor $^{A} /$ nor & $-20,220^{* *}$ & $-13,785^{* *}$ & $-14,117$ & $3,50^{* *}$ \\
\hline $\mathrm{T} 8-\mathrm{T} 3$ & Dupla combinação nor $^{+} /$nor $^{A}$ rin $^{+} /$rin & $-6,045$ & $-11,503^{* *}$ & $-24,467^{*}$ & $2,22^{* *}$ \\
\hline $\mathrm{T} 9-\mathrm{T} 3$ & Dupla combinação nor $^{+} /$nor rin $^{+} /$rin & $-16,830^{* *}$ & $-11,701^{* *}$ & $-20,600$ & $2,38^{* *}$ \\
\hline $\mathrm{T} 7+\mathrm{T} 3-\mathrm{T} 5-\mathrm{T} 4$ & Não-aditividade entre nor $^{+} /$nor $^{A}$ e nor $^{+} /$nor & $-10,591$ & $-3,421$ & 27,400 & $3,02^{* *}$ \\
\hline $\mathrm{T} 8+\mathrm{T} 3-\mathrm{T} 4-\mathrm{T} 6$ & Não-aditividade entre nor $^{+} /$nor $^{A}$ e $\operatorname{rin}^{+} /$rin & 15,246 & 4,420 & 14,267 & 1,15 \\
\hline $\mathrm{T} 9+\mathrm{T} 3-\mathrm{T} 5-\mathrm{T} 6$ & Não-aditividade entre nor $^{+} /$nor e $\mathrm{rin}^{+} / \mathrm{rin}$ & 8,950 & 4,368 & 20,983 & 0,30 \\
\hline
\end{tabular}

${ }^{(1)}$ Médias seguidas da mesma letra não diferem entre si pelo teste de Tukey a $5 \%$ de probabilidade. ${ }^{*} \mathrm{e}{ }^{* *}$ Significativo a $5 \%$ e a $1 \%$ de probabilidade, respectivamente, pelo teste $\mathrm{F}$. 
não-aditividade entre os alelos em heterozigose $\left(\right.$ nor $^{A} \mathrm{e}$ nor), $\left(\right.$ nor $^{A}$ e rin) e (nor e rin) tiveram suas estimativas não significativas para firmeza inicial e meia-vida da firmeza (Tabela 2), o que indica que os efeitos desses locos se somam, quando combinados em um mesmo genótipo.

Quanto ao número de dias decorridos, desde a colheita até os frutos atingirem a firmeza crítica de $2 \times 10^{4} \mathrm{~N} \mathrm{~m}^{-2}$, o híbrido de genótipo nor $^{+} /$nor $^{A}$ não diferiu, significativamente, do genótipo normal nor+/nor ${ }^{+}$(Tabela 2). Esse resultado discorda dos trabalhos de Vilas Boas et al. (1999), Souza et al. (2001), Dias et al. (2003) e Faria et al. (2003), que relataram aumento no tempo de vida pós-colheita dos frutos $n r^{+} /$nor $^{A}$, embora tenham utilizado backgrounds distintos do empregado nesse trabalho. Os genótipos nor ${ }^{+} /$ nor e $\mathrm{rin}^{+} /$rin isolados apresentaram efeitos significativos, tendo aumentado em 2 dias e 1,7 dia, respectivamente, o período para que os frutos atingissem a firmeza de $2 \times 10^{4} \mathrm{~N} \mathrm{~m}^{-2}$ (Tabela 2).
O background do híbrido comercial Carmen $\mathrm{F}_{1}$ foi responsável pelo atraso na perda de firmeza dos frutos, em relação ao background híbrido FloraDade x Tropic (Tabela 2). Assim, confirma-se que a característica longa-vida de um híbrido é devida não somente à utilização do mutante de amadurecimento, mas também a um background genotípico que favorece maior firmeza dos frutos.

Houve efeitos significativos favoráveis das duplas combinações heterozigotas, entre os alelos nor ${ }^{A}$, nor e rin, em desacelerar a perda de firmeza dos frutos (Tabela 2). A dupla combinação nor $^{+} /$nor $^{A} \mathrm{rin}^{+} /$rin apresentou desvios no seu comportamento, em relação à soma dos seus efeitos individuais, conforme acusa a estimativa do contraste que avalia a não-aditividade dos efeitos entre esses locos: o desvio observado foi de 3,5 dias, para os frutos terem sua firmeza reduzida a $2 \times 10^{4} \mathrm{~N} \mathrm{~m}^{-2}$ (Tabela 2). Não foi verificado efeito significativo da heterose no background normal

Tabela 2. Valores médios $\left(10^{4} \mathrm{~N} \mathrm{~m}^{-2}\right)$ da firmeza dos frutos no estádio breaker de amadurecimento, meia-vida (dias) da firmeza, período (dias) para os frutos atingirem firmeza crítica de $2 \times 10^{4} \mathrm{~N} \mathrm{~m}^{-2}$, período médio (dias) estimado, após a colheita no estádio breaker, para os frutos atingirem nota 5 de coloração e contrastes não-ortogonais de interesse entre dez genótipos de tomateiro ${ }^{(1)}$.

\begin{tabular}{|c|c|c|c|c|c|}
\hline Tratamento & Genótipo & $\begin{array}{c}\text { Firmeza } \\
\text { dos frutos } \\
\text { no estádio } \\
\text { breaker }\end{array}$ & $\begin{array}{l}\text { Meia-vida } \\
\text { da firmeza }\end{array}$ & $\begin{array}{c}\text { Período para } \\
\text { atingir } \\
\text { firmeza } \\
\text { crítica }\end{array}$ & $\begin{array}{l}\text { Período para } \\
\text { atingir nota } 5 \\
\text { de coloração }\end{array}$ \\
\hline T1 - FloraDade & nor $^{+} /$nor $^{+} \operatorname{rin}^{+} /$rin $^{+}(=$normal $)$ & $5,197 \mathrm{ab}$ & 9,4 cde & $12,9 \mathrm{cde}$ & $7,3 \mathrm{c}$ \\
\hline T2 - Tropic & nor $^{+} /$nor $^{+} \operatorname{rin}^{+} / \operatorname{rin}^{+}(=$normal $)$ & $3,903 \mathrm{C}$ & $9,6 \mathrm{bcde}$ & $9,0 \mathrm{f}$ & $7,0 \mathrm{c}$ \\
\hline $\mathrm{T} 3-\mathrm{F}_{1}$ (FloraDade $\mathrm{x}$ Tropic) & nor $^{+} /$nor $^{+} \operatorname{rin}^{+} / \operatorname{rin}^{+}(=$normal $)$ & $4,831 \mathrm{~b}$ & $8,7 \mathrm{e}$ & $11,1 \mathrm{def}$ & $7,0 \mathrm{c}$ \\
\hline $\mathrm{T} 4-\mathrm{F}_{1}$ (TOM -610 x FloraDade) & nor $^{+} /$nor $^{A} \operatorname{rin}^{+} /$rin $^{+}$ & $4,605 b c$ & $9,0 \mathrm{de}$ & $10,7 \mathrm{ef}$ & $7,3 \mathrm{c}$ \\
\hline T5 - $F_{1}$ (TOM $-613 \times$ Tropic) & nor $^{+} /$nor rin $^{+} /$rin $^{+}$ & $4,817 b$ & $10,4 \mathrm{abcde}$ & $13,1 \mathrm{~cd}$ & $9,3 \mathrm{abc}$ \\
\hline T6 - $F_{1}$ (TOM -619 x FloraDade) & nor $^{+} /$nor $^{+} \operatorname{rin}^{+} /$rin & $4,497 b c$ & $10,9 \mathrm{abcd}$ & $12,7 \mathrm{cde}$ & $8,0 \mathrm{bc}$ \\
\hline $\mathrm{T} 7-\mathrm{F}_{1}(\mathrm{TOM}-613 \times \mathrm{TOM}-610)$ & nor $/$ nor $\operatorname{rin}^{+} / \mathrm{rin}^{+}$ & $4,633 \mathrm{bc}$ & $11,1 \mathrm{abc}$ & $13,4 \mathrm{~cd}$ & $11,5 \mathrm{ab}$ \\
\hline T8 - $F_{1}(\mathrm{TOM}-619 \times \mathrm{TOM}-559)$ & nor $^{+} /$nor $^{A} \mathrm{rin}^{+} / \mathrm{rin}$ & $4,993 b$ & $12,1 \mathrm{a}$ & $15,9 \mathrm{ab}$ & $12,5 \mathrm{a}$ \\
\hline T9 - $\mathrm{F}_{1}(\mathrm{TOM}-619 \times \mathrm{TOM}-613)$ & nor $^{+} /$nor $\quad \mathrm{rin}^{+} /$rin & $4,737 \mathrm{bc}$ & $12,2 \mathrm{a}$ & $14,8 \mathrm{bc}$ & $12,8 \mathrm{a}$ \\
\hline $\mathrm{T} 10$ - Carmen $\mathrm{F}_{1}$ & nor $^{+} /$nor $^{+}$rin $^{+} /$rin & $5,883 \mathrm{a}$ & $11,5 \mathrm{ab}$ & $17,7 \mathrm{a}$ & $7,8 \mathrm{c}$ \\
\hline Contraste de interesse & Efeito avaliado & \multicolumn{4}{|c|}{------------ Estimativas dos contrastes ------------- } \\
\hline $\mathrm{T} 1-\mathrm{T} 2$ & FloraDade $\mathrm{x}$ Tropic & $1,293^{* *}$ & $-0,1$ & $3,9^{* *}$ & 0,3 \\
\hline $\mathrm{T} 3-[0,5(\mathrm{~T} 1+\mathrm{T} 2)]$ & Heterose no background normal & 0,282 & $-0,8$ & 0,2 & $-0,2$ \\
\hline $\mathrm{T} 4-\mathrm{T} 3$ & Apenas $n o r^{+} /$nor $^{A}$ & $-0,227$ & 0,2 & $-0,4$ & 0,3 \\
\hline $\mathrm{T} 5-\mathrm{T} 3$ & Apenas nor $^{+} /$nor & $-0,015$ & $1,6^{* *}$ & $2,0^{* *}$ & $2,3^{*}$ \\
\hline $\mathrm{T} 6-\mathrm{T} 3$ & Apenas $\mathrm{rin}^{+} / \mathrm{rin}$ & 0,335 & $2,2^{* *}$ & $1,7^{*}$ & 1,0 \\
\hline $\mathrm{T} 7-\mathrm{T} 3$ & Dupla combinação nor $^{A} /$ nor & $-0,198$ & $2,4^{* *}$ & $2,3^{* *}$ & $4,5^{* *}$ \\
\hline $\mathrm{T} 8-\mathrm{T} 3$ & Dupla combinação nor $^{+} /$nor $^{A} \operatorname{rin}^{+} /$rin & 0,162 & $3,3^{* *}$ & $4,8^{* *}$ & $5,5^{* *}$ \\
\hline T9 - T3 & Dupla combinação nor $^{+} /$nor rin $^{+} /$rin & 0,095 & $3,4^{* *}$ & $3,8^{* *}$ & $5,8^{* *}$ \\
\hline $\mathrm{T} 7+\mathrm{T} 3-\mathrm{T} 5-\mathrm{T} 4$ & Não-aditividade entre nor $^{+} /$nor $^{A}$ e nor $^{+} /$nor & 0,043 & 0,6 & 0,7 & 1,8 \\
\hline $\mathrm{T} 8+\mathrm{T} 3-\mathrm{T} 4-\mathrm{T} 6$ & Não-aditividade entre nor $^{+} /$nor $^{A}$ e rin $^{+} /$rin & 0,723 & 0,9 & $3,5^{* *}$ & $4,2^{*}$ \\
\hline $\mathrm{T} 9+\mathrm{T} 3-\mathrm{T} 5-\mathrm{T} 6$ & Não-aditividade entre nor $^{+} /$nor ${\mathrm{e} \mathrm{rin}^{+} / \mathrm{rin}}$ & 0,255 & $-0,4$ & 0,1 & 2,5 \\
\hline
\end{tabular}

${ }^{(1)}$ Médias seguidas da mesma letra não diferem entre si pelo teste de Tukey a $5 \%$ de probabilidade. ${ }^{*}$ e ${ }^{* *}$ Significativo a $5 \%$ e a $1 \%$ de probabilidade, respectivamente, pelo teste $\mathrm{F}$. 
FloraDade x Tropic, para as características relacionadas à firmeza dos frutos.

De maneira geral, os alelos nor ${ }^{A}$, nor e rin, isoladamente ou em duplas combinações heterozigóticas, atuaram para atrasar a síntese de pigmentos dos frutos (Tabela 2). O genótipo nor $^{+} /$nor, quando comparado ao genótipo isogênico normal, apresentou efeito mais drástico sobre a evolução da coloração dos frutos, tendo promovido atraso médio significativo de 2,3 dias, para que os frutos atingissem o valor 5 na escala de notas. Os efeitos dos alelos nor ${ }^{A}$ ou rin foram potencializados, quando em combinações entre si e com nor (Tabela 2). Freitas et al. (1998), Souza et al. (2001), Araújo et al. (2002), e Faria et al. (2003), ao utilizar backgrounds distintos do utilizado neste trabalho, relataram que o mutante nor ${ }^{A}$ em heterozigose, isoladamente, foi res- ponsável pela evolução mais lenta da coloração dos frutos.

A estimativa do contraste que avalia a não-aditividade, entre os efeitos dos locos nor ${ }^{+} /$nor $^{A}$ e $\operatorname{rin}^{+} /$rin, acusou um atraso significativo de 4,2 dias do duplo heterozigoto nor $^{+} /$nor $^{A}$ rin $^{+} /$rin, em relação à soma dos efeitos individuais desses locos, para que os frutos atingissem a nota 5 de coloração (Tabela 2). As estimativas do contraste que avalia a não-aditividade entre os efeitos de nor $^{+} /$nor e nor $^{+} /$nor $^{A}$ foi não significativa, o que indica que os efeitos desses locos, quando combinados em um mesmo genótipo, não apresentaram desvios significativos sobre essa característica, em relação à soma dos efeitos individuais desses locos.

Com o avanço do processo de amadurecimento, constatou-se redução no teor de clorofila, concomitante ao aumento no teor de licopeno e beta-caroteno (Tabela 3).

Tabela 3. Teores médios (mg $100 \mathrm{~g}^{-1}$ ) de clorofila, licopeno e beta-caroteno, dos frutos de dez genótipos de tomateiro, em três estádios de amadurecimento(1).

\begin{tabular}{|c|c|c|c|c|c|}
\hline Tratamento & $\begin{array}{c}\text { Estádio de } \\
\text { amadurecimento }\end{array}$ & Genótipo & Clorofila & Licopeno & Beta-caroteno \\
\hline T1 - FloraDade & Breaker & nor $^{+} /$nor $^{+} \operatorname{rin}^{+} /$rin $^{+}$(= normal) & $1,52 \mathrm{bcde}$ & 57,89hij & $54,50 \mathrm{ijklm}$ \\
\hline T2 - Tropic & Breaker & nor $^{+} /$nor $^{+} \operatorname{rin}^{+} /$rin $^{+}$(= normal) & $1,51 \mathrm{bcde}$ & $31,89 \mathrm{hij}$ & $50,93 \mathrm{klm}$ \\
\hline $\mathrm{T} 3-\mathrm{F}_{1}$ (FloraDade $\mathrm{x}$ Tropic) & Breaker & nor $^{+} /$nor $^{+} \operatorname{rin}^{+} /$rin $^{+}$(= normal) & $1,98 \mathrm{a}$ & $30,23 \mathrm{hij}$ & $55,00 \mathrm{hijkl}$ \\
\hline $\mathrm{T} 4-\mathrm{F}_{1}(\mathrm{TOM}-610 \times$ FloraDade $)$ & Breaker & nor $^{+} /$nor $^{A} \operatorname{rin}^{+} / \operatorname{rin}^{+}$ & $1,52 \mathrm{bcde}$ & $23,03 \mathrm{ij}$ & $51,95 \mathrm{jklm}$ \\
\hline T5 - $\mathrm{F}_{1}$ (TOM -613 x Tropic) & Breaker & nor $^{+} /$nor $\operatorname{rin}^{+} / \mathrm{rin}^{+}$ & $1,32 \mathrm{defg}$ & $24,92 \mathrm{hij}$ & 57,90fghijkl \\
\hline T6 - $\mathrm{F}_{1}(\mathrm{TOM}-619 \times$ FloraDade $)$ & Breaker & nor $^{+} /$nor $^{+}$rin $^{+} /$rin & $1,83 \mathrm{ab}$ & $23,70 \mathrm{ij}$ & $42,04 \mathrm{~m}$ \\
\hline $\mathrm{T} 7-\mathrm{F}_{1}(\mathrm{TOM}-613 \times \mathrm{TOM}-610)$ & Breaker & nor ${ }^{A} /$ nor $\operatorname{rin}^{+} / \mathrm{rin}^{+}$ & $1,67 \mathrm{abc}$ & $28,18 \mathrm{hij}$ & 61,87 efghijk \\
\hline T8 - F 1 (TOM -619 x TOM -559) & Breaker & nor $^{+} /$nor $^{A} \operatorname{rin}^{+} /$rin & $1,59 \mathrm{bcd}$ & $20,28 \mathrm{j}$ & 57,08ghijkl \\
\hline T9 - $\mathrm{F}_{1}(\mathrm{TOM}-619 \times \mathrm{TOM}-613$ & Breaker & nor ${ }^{+} /$nor $\operatorname{rin}^{+} /$rin & $1,44 \mathrm{cdef}$ & $19,84 j$ & 56,73 ghijkl \\
\hline $\mathrm{T} 10$ - Carmen $\mathrm{F}_{1}$ & Breaker & nor $^{+} /$nor $^{+}$rin $^{+} /$rin & 1,52 bcde & 32,98hij & $46,931 \mathrm{~m}$ \\
\hline T1 - FloraDade & Intermediário & nor $^{+} /$nor $^{+} \operatorname{rin}^{+} /$rin $^{+}(=$normal $)$ & $1,24 \mathrm{efgh}$ & $106,46 \mathrm{fgh}$ & $71,57 \mathrm{bcde}$ \\
\hline T2 - Tropic & Intermediário & nor $^{+} /$nor $^{+} \operatorname{rin}^{+} / \operatorname{rin}^{+}(=$normal $)$ & 1,12fghi & 91,01ghij & 67,42 cdefgh \\
\hline T3 $-\mathrm{F}_{1}$ (FloraDade $\mathrm{x}$ Tropic) & Intermediário & nor $^{+} /$nor $^{+} \operatorname{rin}^{+} /$rin $^{+}(=$normal $)$ & 1,13fghi & 105,04 fghi & 64,93defghi \\
\hline $\mathrm{T} 4-\mathrm{F}_{1}(\mathrm{TOM}-610 \times$ FloraDade $)$ & Intermediário & nor $^{+} /$nor $^{A} \operatorname{rin}^{+} / \operatorname{rin}^{+}$ & 1,01ghij & $71,73 \mathrm{hij}$ & $67,95 \mathrm{cdefg}$ \\
\hline T5 - $F_{1}($ TOM -613 x Tropic) & Intermediário & nor $^{+} /$nor $\operatorname{rin}^{+} /$rin $^{+}$ & $0,74 \mathrm{jkl}$ & $68,22 \mathrm{hij}$ & 63,06defghijk \\
\hline T6 - $\mathrm{F}_{1}(\mathrm{TOM}-619 \times$ FloraDade $)$ & Intermediário & nor $^{+} /$nor $^{+}$rin $^{+} /$rin & $1,13 \mathrm{fgh}$ & $75,14 \mathrm{hij}$ & $67,82 \mathrm{cdefgh}$ \\
\hline T7 - $F_{1}(\mathrm{TOM}-613 \times \mathrm{TOM}-610$ & Intermediário & nor $^{A} /$ nor rin $^{+} /$rin $^{+}$ & $0,76 \mathrm{jkl}$ & 63,71 hij & $68,74 \mathrm{cdefg}$ \\
\hline $\mathrm{T} 8-\mathrm{F}_{1}(\mathrm{TOM}-619 \times \mathrm{TOM}-559$ & Intermediário & nor $^{+} /$nor $^{A} \operatorname{rin}^{+} /$rin & 1,02ghij & $76,30 \mathrm{hij}$ & 65,69defghi \\
\hline T9 - $F_{1}($ TOM $-619 \times$ TOM -613) & Intermediário & nor $^{+} /$nor $\operatorname{rin}^{+} /$rin & 0,89hijk & 75,47hij & 64,39defghij \\
\hline $\mathrm{T} 10$ - Carmen $\mathrm{F}_{1}$ & Intermediário & nor $^{+} /$nor $^{+}$rin $^{+} /$rin & 1,12fghi & 90,93ghij & $70,42 \mathrm{bcdef}$ \\
\hline T1 - FloraDade & Maduro & nor $^{+} /$nor $^{+} \operatorname{rin}^{+} / \operatorname{rin}^{+}(=$normal $)$ & $0,60 \mathrm{kl}$ & $430,16 \mathrm{a}$ & 70,20 bcdef \\
\hline T2 - Tropic & Maduro & nor $^{+} /$nor $^{+} \operatorname{rin}^{+} / \operatorname{rin}^{+}(=$normal $)$ & $0,78 \mathrm{jkl}$ & $329,37 \mathrm{bc}$ & 71,33 bcde \\
\hline $\mathrm{T} 3-\mathrm{F}_{1}$ (FloraDade $\mathrm{x}$ Tropic) & Maduro & nor $^{+} /$nor $^{+} \operatorname{rin}^{+} / \operatorname{rin}^{+}(=$normal $)$ & 0,481 & $329,37 \mathrm{bc}$ & 74,61abcde \\
\hline $\mathrm{T} 4-\mathrm{F}_{1}(\mathrm{TOM}-610 \times$ FloraDade $)$ & Maduro & nor $^{+} /$nor $^{A} \operatorname{rin}^{+} / \operatorname{rin}^{+}$ & $0,60 \mathrm{kl}$ & $360,80 \mathrm{ab}$ & $84,43 a$ \\
\hline $\mathrm{T} 5-\mathrm{F}_{1}(\mathrm{TOM}-613 \times \mathrm{Tropic})$ & Maduro & nor ${ }^{+} /$nor $\operatorname{rin}^{+} / \operatorname{rin}^{+}$ & $0,61 \mathrm{kl}$ & $314,88 \mathrm{bc}$ & $75,86 \mathrm{abcd}$ \\
\hline T6 - $\mathrm{F}_{1}(\mathrm{TOM}-619 \times$ FloraDade $)$ & Maduro & nor $^{+} /$nor $^{+}$rin $^{+} /$rin & 0,79ijkl & $166,40 \mathrm{efg}$ & $73,19 \mathrm{abcde}$ \\
\hline T7 - $F_{1}(\mathrm{TOM}-613 \times$ TOM- 610 & Maduro & nor $^{A} /$ nor $\operatorname{rin}^{+} /$rin $^{+}$ & $0,73 \mathrm{jkl}$ & $267,18 \mathrm{~cd}$ & $82,87 \mathrm{ab}$ \\
\hline T8 - $F_{1}(\mathrm{TOM}-619$ x TOM-559) & Maduro & $\operatorname{nor}^{+} / \operatorname{nor}^{A} \operatorname{rin}^{+} /$rin & $0,66 \mathrm{kl}$ & $272,68 \mathrm{~cd}$ & 74,70abcd \\
\hline T9 - F 1 (TOM -619 x TOM - 619) & Maduro & nor $^{+} /$nor $\mathrm{rin}^{+} /$rin & 0,521 & $181,32 \mathrm{ef}$ & $80,16 a b c$ \\
\hline $\mathrm{T} 10$ - Carmen $\mathrm{F}_{1}$ & Maduro & nor $^{+} /$nor $^{+} \operatorname{rin}^{+} /$rin & $0,70 \mathrm{jk} 1$ & $223,96 \mathrm{de}$ & 66,51 defghi \\
\hline
\end{tabular}

(1)Médias seguidas da mesma letra não diferem entre si pelo teste de Tukey a 5\% de probabilidade. 
Esse resultado concorda com os obtidos por Vilas Boas et al. (1999). Os alelos mutantes em heterozigose, isoladamente ou em combinações duplas, tenderam a reduzir o teor de clorofila dos frutos nos estádios breaker e intermediário de maturação (Tabelas 3 e 4), entretanto, esse fato foi revertido no estádio maduro. Nessa fase, nos frutos portadores dos alelos mutantes, a clorofila apresentou teores mais altos e, conseqüentemente, a síntese de licopeno ainda não havia atingido seu ápice. O resultado foi o atraso na pigmentação dos frutos.

Notou-se uma tendência dos alelos mutantes em heterozigose, isoladamente ou em combinações duplas, em reduzir o teor de licopeno nos frutos (Tabela 4), o que está relacionado com o atraso na coloração. No estádio maduro, foi verificado efeito significativo do genótipo $\mathrm{rin}^{+} /$rin, isoladamente, bem como dos genótipos portadores das duplas combinações heterozigóticas entre os três alelos, em estudo sobre o teor de licopeno nos frutos (Tabela 4). Os genótipos $\mathrm{rin}^{+} / \mathrm{rin}$ e $\mathrm{nor}^{+} /$nor $\mathrm{rin}^{+} /$rin foram os que apresentaram redução mais acentuada no teor de licopeno, nos frutos maduros, quando comparados ao genótipo normal (Tabela 4). As estimativas dos contrastes que medem a nãoaditividade entre os efeitos dos locos (nor ${ }^{+} /$nor e nor $^{+}$/ nor $\left.^{A}\right)$ e $\left(\right.$ nor $^{+} /$nor $^{A}$ e rin $^{+} /$rin $)$, sobre o teor de licopeno nos frutos maduros, foram significativas (Tabela 4), o que indica que os genótipos duplo-mutantes, para esses locos, foram menos drásticos em diminuir o teor de licopeno nos frutos, do que seria esperado pela soma dos efeitos dos genótipos portadores desses locos isoladamente.

Tabela 4. Contrastes não-ortogonais de interesse, entre dez genótipos de tomateiro, em três estádios de amadurecimento dos frutos: teores médios de clorofila, licopeno e beta-caroteno.

\begin{tabular}{|c|c|c|c|c|c|}
\hline \multirow{2}{*}{$\begin{array}{l}\text { Contraste de } \\
\text { interesse }\end{array}$} & \multirow{2}{*}{$\begin{array}{c}\text { Estádio de } \\
\text { amadurecimento }\end{array}$} & \multirow[t]{2}{*}{ Efeito avaliado } & \multicolumn{2}{|c|}{ Estimativas dos contrastes } & \multirow{2}{*}{$\frac{\left(\mathrm{mg} 100 \mathrm{~g}^{-1}\right)}{\text { Beta-caroteno }}$} \\
\hline & & & Clorofila & Licopeno & \\
\hline $\mathrm{T} 1-\mathrm{T} 2$ & Breaker & FloraDade x Tropic & 0,003 & 25,99 & 3,57 \\
\hline $\mathrm{T} 3-[0,5(\mathrm{~T} 1+\mathrm{T} 2)]$ & Breaker & Heterose no background normal & $0,466^{* *}$ & $-14,66$ & 2,28 \\
\hline $\mathrm{T} 4-\mathrm{T} 3$ & Breaker & Apenas $n o r^{+} /$nor $^{A}$ & $-0,461^{* *}$ & $-7,19$ & $-3,05$ \\
\hline $\mathrm{T} 5-\mathrm{T} 3$ & Breaker & Apenas nor $^{+} /$nor & $-0,659^{* *}$ & $-5,31$ & 2,90 \\
\hline T6 - T3 & Breaker & Apenas $\mathrm{rin}^{+} / \mathrm{rin}$ & $-0,156$ & $-6,53$ & $-12,96^{* *}$ \\
\hline $\mathrm{T} 7-\mathrm{T} 3$ & Breaker & Dupla combinação nor $^{A} /$ nor & $-0,316^{* *}$ & $-2,05$ & $6,87^{*}$ \\
\hline $\mathrm{T} 8-\mathrm{T} 3$ & Breaker & Dupla combinação nor $^{+} /$nor $^{A} \operatorname{rin}^{+} /$rin & $-0,396^{* *}$ & $-9,95$ & 2,08 \\
\hline T9 - T3 & Breaker & Dupla combinação nor $^{+} /$nor $\operatorname{rin}^{+} /$rin & $-0,539^{* *}$ & $-10,39$ & 1,73 \\
\hline $\mathrm{T} 7+\mathrm{T} 3-\mathrm{T} 5-\mathrm{T} 4$ & Breaker & Não-aditividade entre nor $^{+} /$nor $^{A}$ e $n o r^{+} /$nor & $0,804^{* *}$ & 10,45 & $7,01^{*}$ \\
\hline $\mathrm{T} 8+\mathrm{T} 3-\mathrm{T} 4-\mathrm{T} 6$ & Breaker & Não-aditividade entre nor $^{+} /$nor $^{A}$ e rin $^{+} /$rin & 0,221 & 3,78 & $18,09^{* *}$ \\
\hline $\mathrm{T} 9+\mathrm{T} 3-\mathrm{T} 5-\mathrm{T} 6$ & Breaker & Não-aditividade entre nor $^{+} /$nor e rin $^{+} /$rin & $0,275^{*}$ & 1,45 & $11,79^{*}$ \\
\hline $\mathrm{T} 1-\mathrm{T} 2$ & Intermediário & FloraDade x Tropic & 0,115 & 15,46 & 4,15 \\
\hline $\mathrm{T} 3-[0,5(\mathrm{~T} 1+\mathrm{T} 2)]$ & Intermediário & Heterose no background normal & $-0,054$ & 6,31 & $-4,56$ \\
\hline $\mathrm{T} 4-\mathrm{T} 3$ & Intermediário & Apenas nor $^{+} /$nor $^{A}$ & $-0,118$ & $-33,31$ & 3,02 \\
\hline $\mathrm{T} 5-\mathrm{T} 3$ & Intermediário & Apenas $n o r+$ nor & $-0,385^{* *}$ & $-36,82$ & $-1,87$ \\
\hline $\mathrm{T} 6-\mathrm{T} 3$ & Intermediário & Apenas $\mathrm{rin}^{+} / \mathrm{rin}$ & 0,009 & $-29,90$ & 2,89 \\
\hline $\mathrm{T} 7-\mathrm{T} 3$ & Intermediário & Dupla combinação nor $^{A} /$ nor & $-0,365^{* *}$ & $-41,33$ & 3,81 \\
\hline $\mathrm{T} 8-\mathrm{T} 3$ & Intermediário & Dupla combinação nor $^{+} /$nor $^{A} \operatorname{rin}^{+} /$rin & $-0,107$ & $-28,74$ & 0,76 \\
\hline T9 - T3 & Intermediário & Dupla combinação nor $^{+} /$nor $\mathrm{rin}^{+} /$rin & $-0,231^{* *}$ & $-29,57$ & $-0,54$ \\
\hline $\mathrm{T} 7+\mathrm{T} 3-\mathrm{T} 5-\mathrm{T} 4$ & Intermediário & Não-aditividade entre nor $^{+} /$nor $^{A}$ e nor $^{+} /$nor & 0,138 & 28,80 & 2,67 \\
\hline $\mathrm{T} 8+\mathrm{T} 3-\mathrm{T} 4-\mathrm{T} 6$ & Intermediário & Não-aditividade entre nor $^{+} /$nor $^{A}$ e rin $^{+} /$rin & 0,003 & 34,46 & $-5,17$ \\
\hline $\mathrm{T} 9+\mathrm{T} 3-\mathrm{T} 5-\mathrm{T} 6$ & Intermediário & Não-aditividade entre nor $^{+} /$nor e rin $^{+} /$rin & 0,144 & 37,16 & $-1,56$ \\
\hline $\mathrm{T} 1-\mathrm{T} 2$ & Maduro & FloraDade x Tropic & $-0,181^{*}$ & $100,78^{* *}$ & $-1,13$ \\
\hline $\mathrm{T} 3-[0,5(\mathrm{~T} 1+\mathrm{T} 2)]$ & Maduro & Heterose no background normal & $-0,210^{* *}$ & $-50,39^{* *}$ & 3,84 \\
\hline $\mathrm{T} 4-\mathrm{T} 3$ & Maduro & Apenas nor $^{+} /$nor $^{A}$ & 0,119 & 31,43 & $9,82^{* *}$ \\
\hline $\mathrm{T} 5-\mathrm{T} 3$ & Maduro & Apenas nor $^{+} /$nor & 0,132 & $-14,49$ & 1,25 \\
\hline $\mathrm{T} 6-\mathrm{T} 3$ & Maduro & Apenas $\mathrm{rin}^{+} / \mathrm{rin}$ & $0,313^{* *}$ & $-162,98^{* *}$ & $-1,42$ \\
\hline $\mathrm{T} 7-\mathrm{T} 3$ & Maduro & Dupla combinação nor $^{A} /$ nor & $0,255^{* *}$ & $-62,19^{* *}$ & $8,26^{*}$ \\
\hline $\mathrm{T} 8-\mathrm{T} 3$ & Maduro & Dupla combinação nor $^{+} /$nor $^{A} \operatorname{rin}^{+} /$rin & $0,182^{*}$ & $-56,70^{* *}$ & 0,09 \\
\hline T9 - T3 & Maduro & Dupla combinação nor $^{+} /$nor $\mathrm{rin}^{+} / \mathrm{rin}$ & 0,039 & $-148,06^{* *}$ & 5,55 \\
\hline $\mathrm{T} 7+\mathrm{T} 3-\mathrm{T} 5-\mathrm{T} 4$ & Maduro & Não-aditividade entre nor $^{+} /$nor $^{A}$ e $n o r^{+} /$nor & 0,004 & $79,13^{* *}$ & $-2,81$ \\
\hline $\mathrm{T} 8+\mathrm{T} 3-\mathrm{T} 4-\mathrm{T} 6$ & Maduro & Não-aditividade entre nor $^{+} /$nor $^{A}$ e rin $^{+} /$rin & $-0,250^{*}$ & $74,85^{*}$ & $-8,31$ \\
\hline $\mathrm{T} 9+\mathrm{T} 3-\mathrm{T} 5-\mathrm{T} 6$ & Maduro & Não-aditividade entre nor $^{+} /$nor e rin $^{+} /$rin $^{-}$ & $-0,407^{* *}$ & 29,41 & 5,73 \\
\hline
\end{tabular}

${ }^{*}$ e ${ }^{* *}$ Significativo a $5 \%$ e a $1 \%$ de probabilidade, respectivamente, pelo teste $\mathrm{F}$. 


\section{Conclusões}

1. Isoladamente, o alelo rin mostra-se mais promissor em prolongar a firmeza dos frutos, quando comparado com o alelo nor.

2. O uso de híbridos heterozigotos, nas duplas combinações entre os locos nor ${ }^{A}$, nor e rin, propicia frutos mais firmes, em comparação com os híbridos portadores desses alelos isoladamente; embora os frutos duplomutantes sofram atraso na evolução da coloração, esse não é um fator limitante.

3. A característica longa-vida de um híbrido é resultante não somente da utilização de mutantes de amadurecimento, mas também do background genotípico.

\section{Agradecimentos}

Ao CNPq/RHAE (Programa de Recursos Humanos em Áreas Estratégicas), pela concessão de bolsas de doutorado; à Faepe/Ufla, pelo apoio institucional; à Fapemig, pelo auxílio financeiro; à Capes/MEC, pela concessão de bolsa; à HortiAgro Sementes Ltda., por disponibilizar seus funcionários para auxílio nas atividades de campo.

\section{Referências}

ARAÚJO, M.L.; MALUF, W.R.; GOMES, L.A.A.; OLIVEIRA, A.C.B. Intra and interlocus interactions between alcobaça (alc), crimson (ogc) and high pigment (hp) loci in tomato Lycopersicon esculentum Mill. Euphytica, v.125, p.215-226, 2002.

BENITES, F.R.G. Estudos genético-fisiológicos dos mutantes, alcobaça (alc), non-ripening (nor) e ripening-inhibitor (rin) em tomateiro. 2003. 106p. Dissertação (Mestrado) - Universidade Federal de Lavras, Lavras.

BRUINSMA, J. The quantitative analysis of chlorophylls A and B in plant extracts. Photochemistry and Photobiology, v.2, p.241249, 1963.

BUESCHER, R.W.; TIGCHELAAR, E.C. Pectinesterase, polygalacturonase, cx-cellulase activities and softening of the rin tomato mutant. HortScience, v.10, p.624-625, 1975.

CALBO, A.G.; NERY, A.A. Medida de firmeza em hortaliças pela técnica de aplanação. Horticultura Brasileira, v.13, p.14-18, 1995. DIAS, T.J.M.; MALUF, W.R.; FARIA, M.V.; FREITAS, J.A.; GOMES, L.A.A.; RESENDE, J.T.V.; AZEVEDO, S.M. Alcobaça allele and genotypic backgrounds affect yield and fruit shelf life of tomato hybrids. Scientia Agricola, v.60, p.269-275, 2003.

FARIA, M.V.; MALUF, W.R.; AZEVEDO, S.M.; ANDRADEJÚNIOR, V.C.; GOMES, L.A.A.; MORETTO, P.; LICURSI, V.
Yield and post-harvest quality of tomato hybrids heterozygous at the loci alcobaça, old gold-crimson or high pigment. Genetics and Molecular Research, v.2, p.317-327, 2003.

FREITAS, J.A. de; MALUF, W.R.; GOMES, L.A.A.; OLIVEIRA, A.C.B. de; MARTINS, W.; BRAGA, R.S. Padrão de amadurecimento e conservação pós-colheita de frutos de tomateiro, em função das diferentes constituições genotípicas no loco alcobaça. Revista Brasileira de Fisiologia Vegetal, v.10, p.191-196, 1998.

KOPELIOVITCH, E.; RABINOWITCH, H.D.; MIZRAHI, Y.; KEDAR, N. The potential of ripening mutants for extending the storage life of the tomato fruit. Euphytica, v.28, p.99-104, 1979.

LOBO, M. Genetic and physiological studies of the "Alcobaça” tomato ripening mutant. 1981. 107p. Thesis (Ph.D.) - University of Florida, Florida.

LOBO, M.; BASSET, M.J.; HANNAH, L.C. Inheritance and characterization of the fruit ripening mutation in 'alcobaça' tomato. Journal of American Society for Horticultural Science, v.109, p.741-745, 1984.

MALUF, W.R.; MIRANDA, J.E.C.; CAMPOS, J.R. Análise genética de um cruzamento dialélico de tomate. I. Características referentes à produção de frutos. Pesquisa Agropecuária Brasileira, v.17, p.633634, 1982.

MUTSCHLER, M.A. Inheritance and linkage of the 'alcobaça' ripening mutant tomato. Journal of American Society for Horticultural Science, v.109, p.500-503, 1984a.

MUTSCHLER, M.A. Ripening and storage characteristics of the 'alcobaça' ripening mutant in tomato. Journal of American Society for Horticultural Science, v.109, p.504-507, 1984b.

MUTSCHLER, M.A.; WOLFE, D.W.; COBB, E.D.; YOURSTONE, K.S. Tomato fruit quality and shelf life in hybrids heterozygous for the alc ripening mutant. HortScience, v.27, p.352355, 1992

NAGATA, M.; YAMASHITA, I. Simple method for simultaneous determination of chlorophyll and carotenoids in tomato fruit. Nippon Shokuhin Kogyo Gakkaishi, v.39, p.925-928, 1992.

SINK, K.C.; HERNER, R.C.; KNOWLTON, L.L. Chlorophyll and carotenoids of the rin tomato mutant. Canadian Journal of Botany, v.52, p.1657-1660, 1974.

SOUZA, J.C.; MALUF, W.R.; SOUZA SOBRINHO, F.; GOMES, L.A.A.; MORETO, P.; LICURSI, V. Características de produção e conservação pós-colheita de frutos de tomateiros híbridos portadores do alelo "alcobaça". Ciência e Agrotecnologia, v.25, p.503-509, 2001.

TIGCHELAAR, E.C.; McGLASSON, W.B.; BUESCHER, R.W. Genetic regulation of tomato fruit ripening. HortScience, v.13, p.508513, 1978

VILAS BOAS, E.V.B.; CHITARRA, A.B.; MALUF, W.R.; CHITARRA, M.I.F. Influência do alelo alcobaça em heterozigose sobre a vida de prateleira e qualidade pós-colheita de tomates. Ciência e Agrotecnologia, v.23, p.650-657, 1999. 